\title{
Growth and Energy Transfer of Newly Discovered Fluorescence in Polystyrene Fine Particles
}

\author{
$\underline{\text { Alex Kuo }}$ \\ Scientist, 83 Borough Drive, Toronto, Ontario, Canada, M1P 5E4. Email: alex1.kuo2@yahoo.com \\ doi:10.5618/chem.2011.v1.n1.6 || Received: 20-08-2011, Accepted: 04-09-2011, Available online: 06-09-2011
}

\begin{abstract}
This is a following-up paper about the newly discovered fluorescence emissions observed in polystyrene fine particles. The details will be discussed regarding how and when these new fluorescence emissions appear, and how energy transfer occurs in different fluorophores. It is found that the fluorescence emissions grow mostly after the first day's sitting under ambient conditions and the first two days of vacuum drying. The fluorescence is still changing after 3 months, indicating polystyrene chains are not in an equilibrium state. The absorption peaks and emission peaks have large overlap that can lead to efficient energy transfer. An attempt is also made to categorize the emission and absorption peaks by the number of phenyl groups in their originating fluorophores.
\end{abstract}

Keywords: fluorescence growth; polystyrene fine particles; energy transfer, phenyl aggregations.

\section{Introduction}

Fluorescence spectroscopy has been widely employed in various fields of science: biology (biochemistry, molecular biophysics, and biomedicine), materials science, mineralogy and gemology etc. It is a powerful tool to probe the microscopic changes in a molecule or a system because of its acute sensitivity to changes of the environmental (such as $\mathrm{pH}$ and electric potential), structural and dynamic properties of molecules. Fluorescence is more sensitive to environment of fluorophores than UV/visible spectroscopy by a factor of $10-10^{5}$ due to the increased time the molecule spends in the excited state. Moreover, there are a few other advantages of fluorescent spectroscopy: high selectivity, nondestructiveness and real-time performance [1].

Fluorescence spectroscopic studies can be carried out at many levels ranging from simple measurement of steady-state fluorescence emission, anisotropy, to timeresolved measurements. Fluorescence signals can be emitted from inherently fluorescent groups, or extrinsic fluorescent labels/probes through incorporation of additives with fluorescent groups. Sometimes intrinsic fluorescence is nonspecific and weak, extrinsic fluorescent probes are required in order to obtain useful information.

Many polymers contain fluorescent constituents, either as side groups attached to the polymeric backbone or as part of backbone. Typically fluorescence occurs from aromatic molecule. Polystyrene (PS), an inexpensive and common polymer in our daily life, is structurally a long hydrocarbon chain, with intrinsic fluorescent phenyl groups attached to every other carbon atom. The fluorescence of polystyrene has been widely studied. Recently I have reported a new luminescence phenomenon observed in the course of solvent evaporation in polystyrene fine particles [2]. A few strong fluorescence emissions in longer wavelength regions than the benzene excimers' emissions were discovered in polystyrene fine particles. This following-up article presents more details about the growth of these fluorescence emissions and the energy transfer between different fluorophores.

\section{Materials and Experimental}

Please refer to Section 2 of [2] about the preparation of polystyrene fine particles and the settings of fluorescence spectrometer.

\section{Results and Discussion}

3.1 Fluorescence growths at different excitation energies. The PS fine particles were allowed to dry freely under ambient conditions for 5 days and then were dried in a vacuum of $\sim 10^{-2}$ Torr for a few months. Their fluorescence was monitored every day in the first 20 days, and then less frequently afterward. To trace the fluorescence growths, ratios of the fluorescence spectra from two successive days or two successive measurements (in the cases of that fluorescence is not monitored every day) at the same excitation energies are plotted and explored. It is found that the fluorescence 
ratios exhibit the greatest changes after the first day's of free solvent evaporation under ambient conditions, and after the first day and the second day of the vacuum drying. The discussion will be focused on the fluorescence growths in PS fine particles in these days.

Fig.1 presents the normalized fluorescence emissions at different excitation energies for as-prepared PS fine particles in the first day. The excitation energies are selected base on the excitation spectra in Fig. 2 of the previous report [2]. The fluorescence emission at $\lambda_{\mathrm{ex}}=$ $260 \mathrm{~nm}$ is a broad peak centered at $330 \mathrm{~nm}$ (black curve). When excited at $278 \mathrm{~nm}$ (red curve), the emission peak becomes less symmetric with a shoulder at $303 \mathrm{~nm}$. The peak centered at $330 \mathrm{~nm}$ is split into two with shoulders at $325 \mathrm{~nm}$ and $339 \mathrm{~nm}$, respectively. Different from the emissions at the excitation energies of $260 \mathrm{~nm}$ and 278 $\mathrm{nm}$, the fluorescence emission profile at $\lambda_{\mathrm{ex}}=296 \mathrm{~nm}$ (green curve) has a small shoulder at $316 \mathrm{~nm}$ besides an obvious shoulder at $325 \mathrm{~nm}$. Moreover, the emission starts to grow sharp from $337 \mathrm{~nm}$ to have a dominant peak with the top at $339 \mathrm{~nm}$ and a shoulder at $\mathbf{3 4 7} \mathrm{nm}$. The fluorescence emission profile at $\lambda_{\mathrm{ex}}=303$ (blue) $\mathrm{nm}$ is similar to that of $278 \mathrm{~nm}$. When excited at $325 \mathrm{~nm}$ (yellow), the emission profile shares some similarity with that at $\lambda_{\mathrm{ex}}=296 \mathrm{~nm}$ except that there are some structured emissions in longer wavelength region than $370 \mathrm{~nm}$. This portion of emission has similar structured bands as the emission at $\lambda_{\mathrm{ex}}=340 \mathrm{~nm}$ (purple). These visible structured bands are located at $372 \mathrm{~nm}, 378 \mathrm{~nm}$, $383 \mathrm{~nm}, 388 \mathrm{~nm}, 394 \mathrm{~nm}, 404 \mathrm{~nm}$, and $419 \mathrm{~nm}$. The fluorescence emission excited at $360 \mathrm{~nm}$ (grey) shows a sharp peak at $376 \mathrm{~nm}$ with a shoulder at $379 \mathrm{~nm}$, and two board peaks centered at $404 \mathrm{~nm}$ and $426 \mathrm{~nm}$, respectively. The fluorescence emission excited at 380 $\mathrm{nm}$ (dark red) shows a sharp peak at $397 \mathrm{~nm}$ with a shoulder at $404 \mathrm{~nm}$, and a broad peak at $426 \mathrm{~nm}$. In the cases at excitation energies of $360 \mathrm{~nm}$ and $380 \mathrm{~nm}$, the first sharp emission peaks might not be a complete peak due to the fact that emission collections start from wavelengths that are very close to the excitation energies. Therefore the peak wavelengths of $376 \mathrm{~nm}$ and $397 \mathrm{~nm}$ are not necessary accurate. By comparison of the fluorescence emissions at different excitation energies, it is clear that there are not only benzene excimer emissions in the as-prepared PS fine particles. The emissions in longer wavelength regions indicate that the fluorophores with two or more interacting phenyl groups have been formed in the as-prepared PS fine particles. As discussed previously [2], the folding of polystyrene chains driven by hydrophobic forces during reprecipitation is responsible for the formations of phenyl aggregations.
After one day's free solvent evaporation under ambient conditions, the PS fine particles generate stronger fluorescence emissions at longer wavelengths, as indicated by the relative stronger fluorescence intensities in longer wavelength regions in Fig.2 than the fluorescence intensities in Fig.1. The fluorescence spectra at different excitation energies are normalized in the same way in Fig.2 and in Fig.1, as well as the spectra presented for other selected days (Figures 4-6, 8). Therefore, a direct comparison of the normalized spectra in different days can clearly show how the fluorescence emissions at different excitation energies grow. In the second day under ambient conditions, the PS fine particles share similarity in their fluorescence emissions at the excitation energy range of $260-303 \mathrm{~nm}$. The fluorescence at $\lambda_{\mathrm{ex}}=340 \mathrm{~nm}$ show the structured emissions in the same wavelengths as those in the asprepared PS fine particles, while fluorescence relative intensities have changed. Based on the emission profiles, the different excitation energies can be categorized into four types: $\lambda_{\mathrm{ex}}=260-303 \mathrm{~nm}(260,278,296$ and $303 \mathrm{~nm})$; $\lambda_{\text {ex }}=325 \mathrm{~nm} ; \lambda_{\text {ex }}=340 \mathrm{~nm} ; \lambda_{\text {ex }}=360$ and $380 \mathrm{~nm}$, which might correspond to different types of fluorophores.

Fig.3 presents the ratios of fluorescence emissions at each excitation energy from the PS particles stayed under ambient conditions for different days: 1) thin curves: the ratios of fluorescence emissions in the second day to those in the first day (as-prepared sample); 2) thick curves: the ratios of fluorescence emissions in the third day to those in the second day. These spectral ratios are more direct measurements for the fluorescence growth in the PS particles, particularly for the growth rate of fluorescence emissions. The growth magnitudes are indicated by the ratios in the y-axis. Any peaks shown in the spectral ratio profiles indicate new emissions or relatively greater fluorescence growth rate for these emissions. A flat straight line indicates the same fluorescence growth rate for all the emissions. In general, the fluorescence growth is much greater in the second day than in the third day, as indicated by the larger ratios presented in the thinner curves. This observation is generally true for all the studied excitation energies. Furthermore, the new fluorescence emissions occurred mostly in the first day's solvent evaporation, as indicated by more and stronger peaks in the ratio profiles shown in thinner curves. Therefore following discussion is focused on the fluorescence growth after the first day of free solvent evaporation when the PS fine particles are left under ambient conditions, i.e., in the $2^{\text {nd }}$ day after the preparation. 


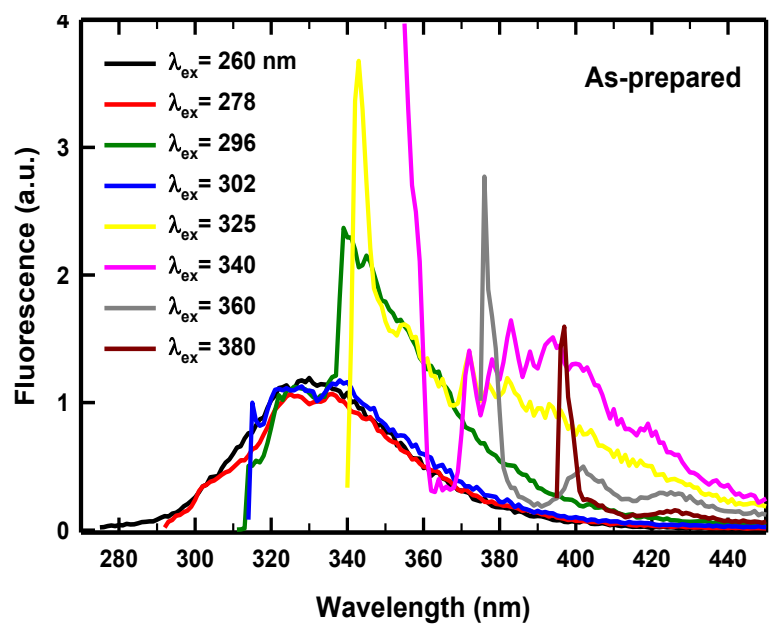

Fig. 1. The normalized fluorescence spectra at different excitation energies from as-prepared PS fine particles.

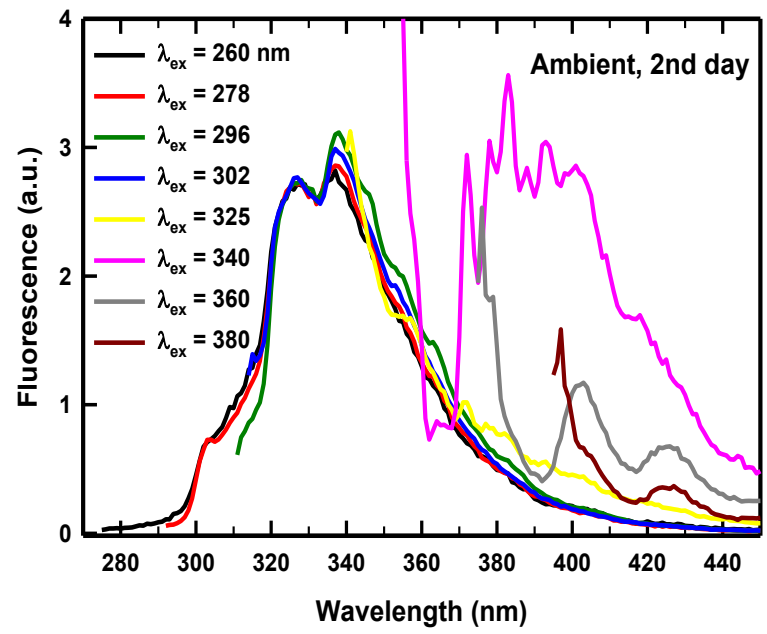

Fig. 2. The normalized fluorescence spectra at different excitation energies from PS fine particles sitting under ambient conditions for one day.

Fig. 3(left). The ratios of fluorescence spectra at different excitation energies: 1) thin curves: emissions from PS fine particles sitting under ambient conditions for one day ratio to those from as-prepared PS fine particles.2) thick curves: emissions from PS fine particles sitting under ambient conditions for two days ratio to those PS fine particles sitting under ambient conditions for one day. (Note; ' $\mathrm{X} 5$ ' means that the $y$-axis of curve is multiplied by a factor of 5.)

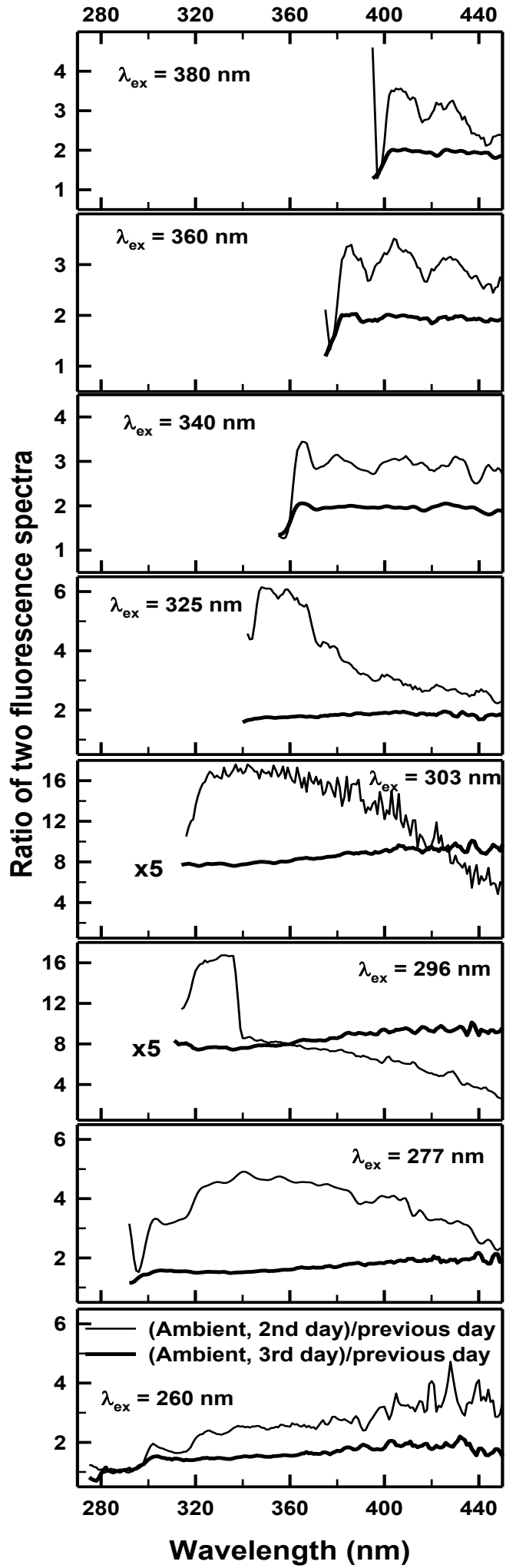


The fluorescence ratio profile at $\lambda_{\text {ex }}=260 \mathrm{~nm}$ reveals that emissions growth/appearance occurs not only at $\mathbf{3 0 3}$ $\mathrm{nm}, 325 \mathrm{~nm}, 339 \mathrm{~nm}$, but also at $347 \mathrm{~nm}, 356 \mathrm{~nm}, 361$ $\mathrm{nm}$ and $383 \mathrm{~nm}$. This indicates that excitation of phenyl monomers at $260 \mathrm{~nm}$ can lead to the emissions of different fluorescence besides excimer's emission at 325 $\mathrm{nm}$ in the PS particles after one day's solvent evaporation under ambient conditions.

The fluorescence ratio profile at $\lambda_{\mathrm{ex}}=278 \mathrm{~nm}$ is a strong broad peak in the region between $300-450 \mathrm{~nm}$ with shoulders at $303 \mathrm{~nm}, 329 \mathrm{~nm}, 339 \mathrm{~nm}, 356 \mathrm{~nm}, \mathbf{3 6 5}$ $\mathrm{nm}, 383 \mathrm{~nm}$ and $404 \mathrm{~nm}$. Compared with the profile at $\lambda_{\mathrm{ex}}=260 \mathrm{~nm}$, some emissions appears to have the same fluorescence wavelengths (such as $303 \mathrm{~nm}, 339 \mathrm{~nm}, 356$ $\mathrm{nm}$ and $383 \mathrm{~nm}$ ), some emissions have been shifted (such as $329 \mathrm{~nm}$ is $4 \mathrm{~nm}$ red shifted from $325 \mathrm{~nm}$ ) or appeared as new emission (404 nm).

When excited at $296 \mathrm{~nm}$, the fluorescence ratio profile of PS particles after a day's free solvent evaporation exhibited a sharp drop between 335.5 and $340 \mathrm{~nm}$, leaving a strong emission between $314 \mathrm{~nm}$ to $335.5 \mathrm{~nm}$ with a maximum located at $329 \mathrm{~nm}$. As seen in the green curves in Fig. 1 and Fig. 2, the sharp drop in the ratio profile is due to that the intensity ratio of two emission peaks at $325 \mathrm{~nm}$ and $339 \mathrm{~nm}$ changes from the $\sim 1 / 2$ to nearly 1. As will be discussed in Section 3.3, this is caused by the energy transfer of the emission at $339 \mathrm{~nm}$ to emissions at longer wavelengths at $347 \mathrm{~nm}$, $365 \mathrm{~nm}$ and so on. The energy transfer results in relatively lower fluorescence intensity at $339 \mathrm{~nm}$.

The fluorescence ratio profile at $\lambda_{\mathrm{ex}}=303 \mathrm{~nm}$ resembles that at $\lambda_{\mathrm{ex}}=278 \mathrm{~nm}$ very much, except that it is less structured and their recorded wavelength ranges are different due to the limitation from the excitation energies.

The fluorescence ratio profile at $\lambda_{\mathrm{ex}}=325 \mathrm{~nm}$ has a peak centered at a shallow dip at $354 \mathrm{~nm}$ between two small shoulders at $350 \mathrm{~nm}$ and $359 \mathrm{~nm}$. Similar to the cases at $\lambda_{\text {ex }}=296$ and $303 \mathrm{~nm}$, the growths of emissions at longer wavelengths have similar rate and the ratios appear as flat lines.

The fluorescence ratio profile at $\lambda_{\mathrm{ex}}=340 \mathrm{~nm}$ shows an interesting observation. The peaks shown in the ratio profile are not exactly corresponding to the fluorescence emission peaks in the purple curves in Figures 1 and 2 except for the first peak centered at $365 \mathrm{~nm}$. The second peak in the ratio profile between $370.5 \mathrm{~nm}$ and $395 \mathrm{~nm}$ covers most of fine structures in the fluorescence emission spectrum. The third peak in the ratio profile is structured peak between $395 \mathrm{~nm}$ and $420 \mathrm{~nm}$. The different growth rates for the emission peaks at different wavelengths indicate that the fluorescence emissions at these wavelengths are from different fluorescence paths and/or fluorophores.

The fluorescence ratio profile at $\lambda_{\mathrm{ex}}=360 \mathrm{~nm}$ has three obvious peaks centered at $385.5 \mathrm{~nm}, 404 \mathrm{~nm}$, and $426 \mathrm{~nm}$, respectively. The latter two peaks correspond to the fluorescence emissions in the same wavelengths, but the first peak corresponds to neither the shoulder emission at $379 \mathrm{~nm}$, nor the sharp peak at $376 \mathrm{~nm}$. This might be due to the fact mentioned previously that the first emission peak is so close to the excitation wavelength that it might not be real or the real peak position might not be detectible. Similarly, the fluorescence ratio profile at $\lambda_{\mathrm{ex}}=380 \mathrm{~nm}$ does show peaks in the same wavelengths as the fluorescence emission peaks at $404 \mathrm{~nm}$ and $426 \mathrm{~nm}$, but not the first sharp emission peak at $397 \mathrm{~nm}$.

Fig. 4 presents the normalized fluorescence spectra recorded on the PS fine particles sitting under ambient conditions for 4 days, i.e., the $5^{\text {th }}$ day, the last day before it was left in a vacuum of $10^{-2}$ Torr for further drying. Compared to the spectra recorded earlier as shown in Figures 1 and 2, it is obvious that the fluorescence emissions in longer wavelength regions become stronger, especially for the fluorescence at the excitation energies of 360 and $380 \mathrm{~nm}$. When the PS fine particles are left in vacuum of $10^{-2}$ Torr for drying for one day, the emissions at longer wavelengths become much stronger, as indicated by their much larger relative intensities shown in their normalized fluorescence spectra presented in Fig. 5. The emissions in longer wavelengths continue to grow a lot after the first two days' vacuum drying, as seen in Fig. 6.

The PS fluorescence growths during vacuum drying are also evaluated via the fluorescence ratio profiles. Fig.7 presents the ratios of fluorescence emissions at each excitation energy from the PS particles after vacuum drying: 1) thin curves: the ratios of fluorescence from the PS particles after 1 day's vacuum drying to the fluorescence before vacuum drying ( $5^{\text {th }}$ day under ambient conditions); 2) thick curves: the ratios of the fluorescence recorded in the second day of vacuum drying to the fluorescence in the first day's vacuum drying. Different from the fluorescence growths under ambient conditions where fluorescence emissions in shorter wavelength regions grow much stronger, the strong fluorescence growths in the first two days of vacuum drying occur in longer wavelength regions. 


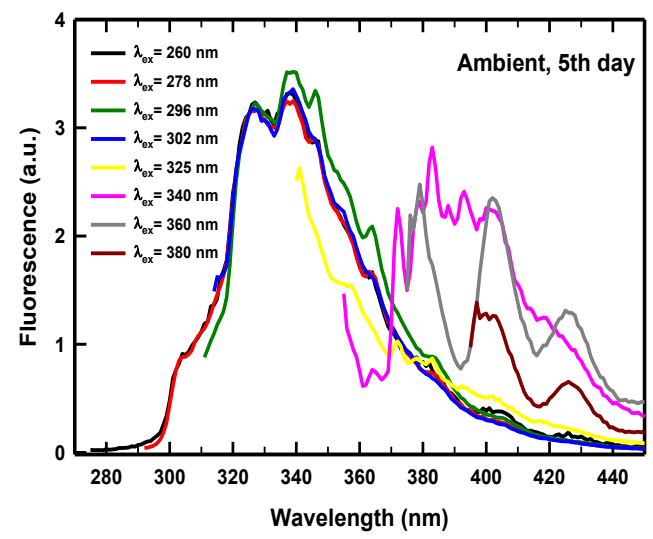

Fig. 4. The normalized fluorescence spectra at different excitation energies from PS fine particles sitting under ambient conditions for 4 days.

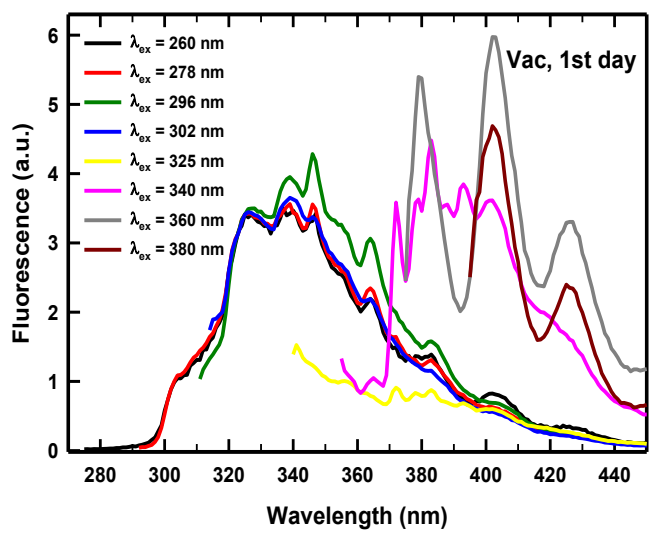

Fig. 5. The normalized fluorescence spectra at different excitation energies from PS fine particles in vacuum for 1 day.

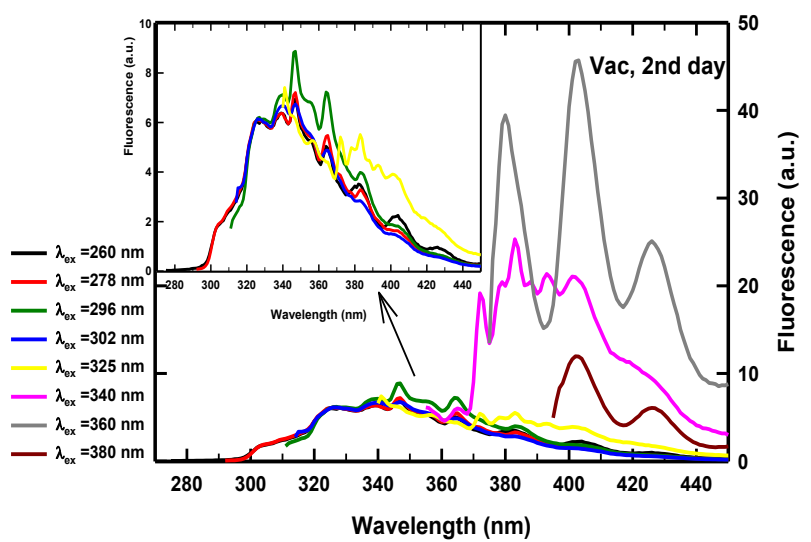

Fig. 6. The normalized fluorescence spectra at different excitation energies from PS fine particles in vacuum for 2 days.

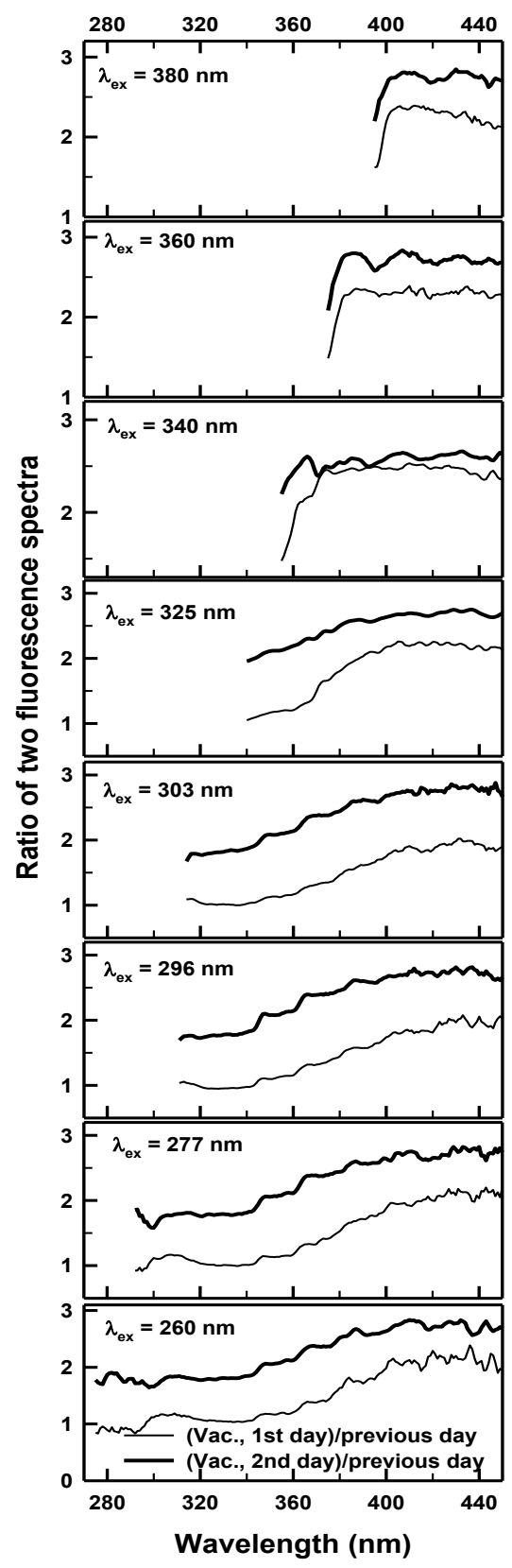

Fig. 7. The ratios of fluorescence spectra at different excitation energies: 1) thin curves: emissions from PS fine particles after one day's vacuum drying to those from PS particles sitting under ambient conditions for 4 days; 2) thick curves: emissions from PS fine particles after two days' vacuum drying to those from PS fine particles after one day's vacuum drying. 
In general, the fluorescence of PS fine particles grow more slowly after the first two days when PS particles are stored in vacuum, but the growth is going continuously and stably with longer drying times. As shown in Fig. 8, after 3 months' vacuum drying, the intensities of fluorescence in longer wavelength regions with the excitation energies at $325,340,360$ and $380 \mathrm{~nm}$ are relatively much stronger than the fluorescence emissions at the other excitation energies between 260-302 nm. This implies that the fluorophorous phenyl aggregations with more phenyl groups are growing. It seems that not only the solvent evaporation but also the time are important factors for the formations of these fluorophores. Interesting, after around 3 months' vacuum drying, the fluorescence ratios to the previous measurements conducted $\sim 2$ weeks prior to this measurement, i.e., the fluorescence growth rates in two weeks exhibits very different profiles, as shown in Fig. 9. The fluorescence emissions at excitation energies 260$303 \mathrm{~nm}$ show stronger growths in the shorter wavelength regions. This resembles the ratio profiles under ambient conditions (Fig. 3) but is different from the profiles in the first two days' vacuum drying (Fig. 7). The most surprising observation is that the ratio profile at $\lambda_{\mathrm{ex}}=360 \mathrm{~nm}$ shows very strong peaks centered at the same wavelengths $(383,404$ and $426 \mathrm{~nm})$ as the fluorescence emissions at the excitation energy of $360 \mathrm{~nm}$, while no similar phenomenon is observed for the other ratio profiles.

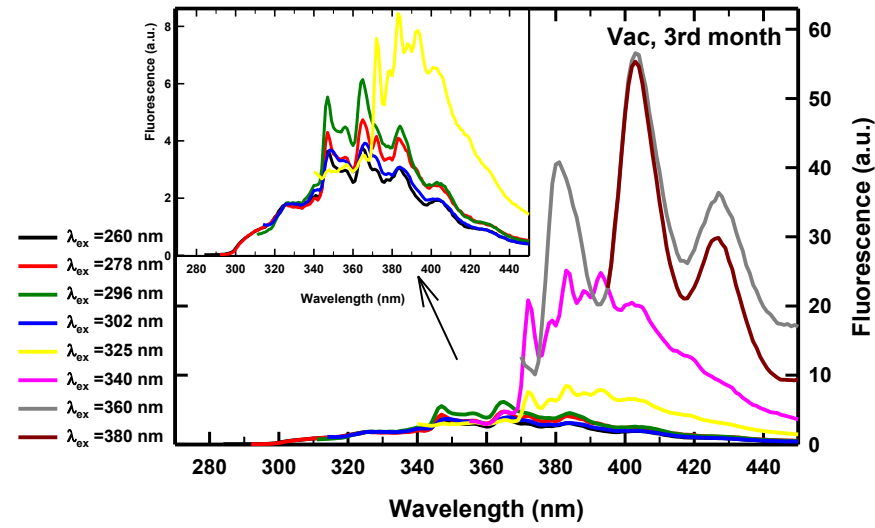

Fig. 8 (above). The normalized fluorescence spectra at different excitation energies from PS fine particles in vacuum for 3 months.

Fig. 9 (right). The ratios of fluorescence spectra at each excitation energy from PS fine particles after 3 months' vacuum drying to the spectra recorded on PS particles 2 weeks before.

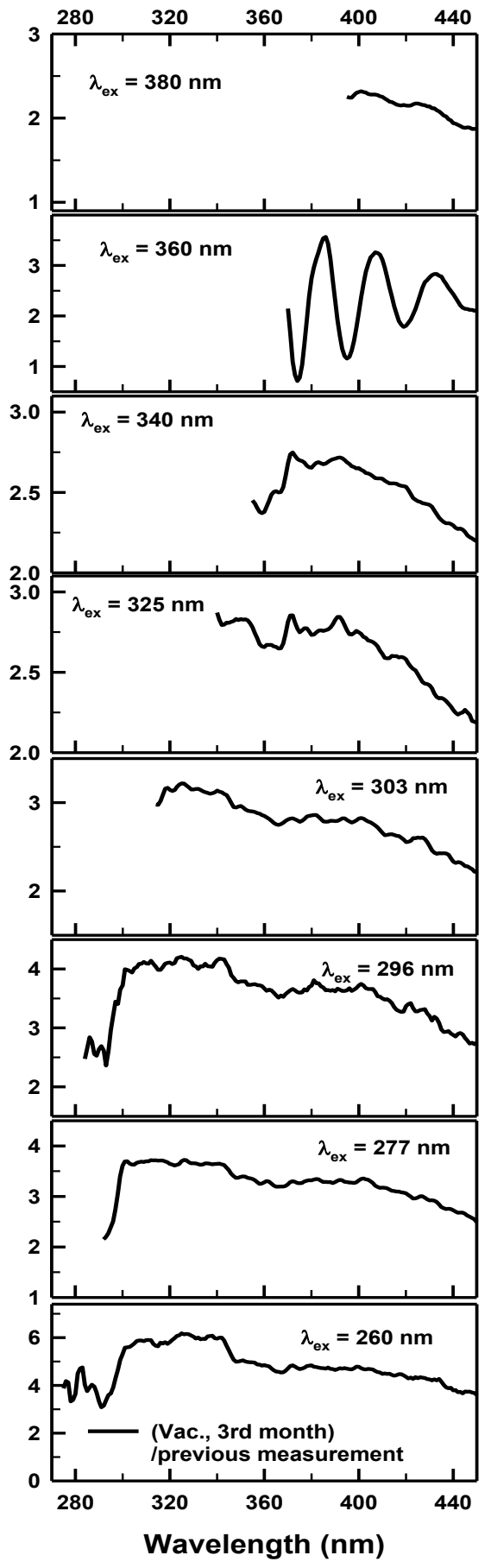

3.2 Growth of excitation/absorption bands at selected fluorescence emissions. Fig. 10 is the excitation spectra of PS fine particles recorded at selected fixed fluorescence emissions in different days under ambient conditions: as-prepared (the $1^{\text {st }}$ day), the $2^{\text {nd }}$ day, and the $3^{\text {rd }}$ day. The excitation spectra at $\lambda_{\mathrm{em}}=325,347$ and 365 $\mathrm{nm}$ are similar. Besides the strong absorption band of benzene at around $260 \mathrm{~nm}$, there are a shoulder peak at $269 \mathrm{~nm}$, a broad peak between 274-304nm with fine structures, and a peak centered at $325 \mathrm{~nm}$ ( $\lambda_{\text {em }}=347$ and $365 \mathrm{~nm}$ only). The excitation spectra at $\lambda_{\mathrm{em}}=383,404$ and $426 \mathrm{~nm}$ also share the same similarity with those at 
$\lambda_{\mathrm{em}}=325,347$ and $365 \mathrm{~nm}$ in their overlapped wavelength ranges. However, in longer wavelength regions, there are some strong absorption bands centered at $325,340,360$ and $380 \mathrm{~nm}$. The similarity of the excitation spectra indicates the high efficiency of energy transfer between different fluorophores. This is because there is large overlap between absorption and emission peaks. For instance, the $325 \mathrm{~nm}$ is not only the wavelength of benzene excimer emission, but also the wavelength of an absorption band's center. The energy transfer will be discussed in more details in Section 3.3.

The spectral ratios of excitation spectra recorded on the as-prepared PS particles and the PS particles after one day's solvent evaporation under ambient conditions at each selected emissions are plotted together in Fig. 11. Not only are the excitation spectra similar at $\lambda_{\mathrm{em}}=325$,
347 and $365 \mathrm{~nm}$, but their spectral ratio profiles at these emissions are also very similar except that the ratio profile at $\lambda_{\mathrm{em}}=365 \mathrm{~nm}$ shows more fine structures (green). The ratio profile at $\lambda_{\text {em }}=404 \mathrm{~nm}$ (grey) has largest magnitude. This indicates that formation of fluorophore (s) and energy transfer paths leading to the emission at $404 \mathrm{~nm}$ grows mostly.

In the third day under ambient conditions, PS fine particles exhibited much slower and similar growth rate for different fluorophores, as indicated by the smaller ratio magnitude and flat curves shown in the ratio profiles in Fig. 12. This is consistent with corresponding small fluorescence growth rate as shown thicker curves in Fig. 3.

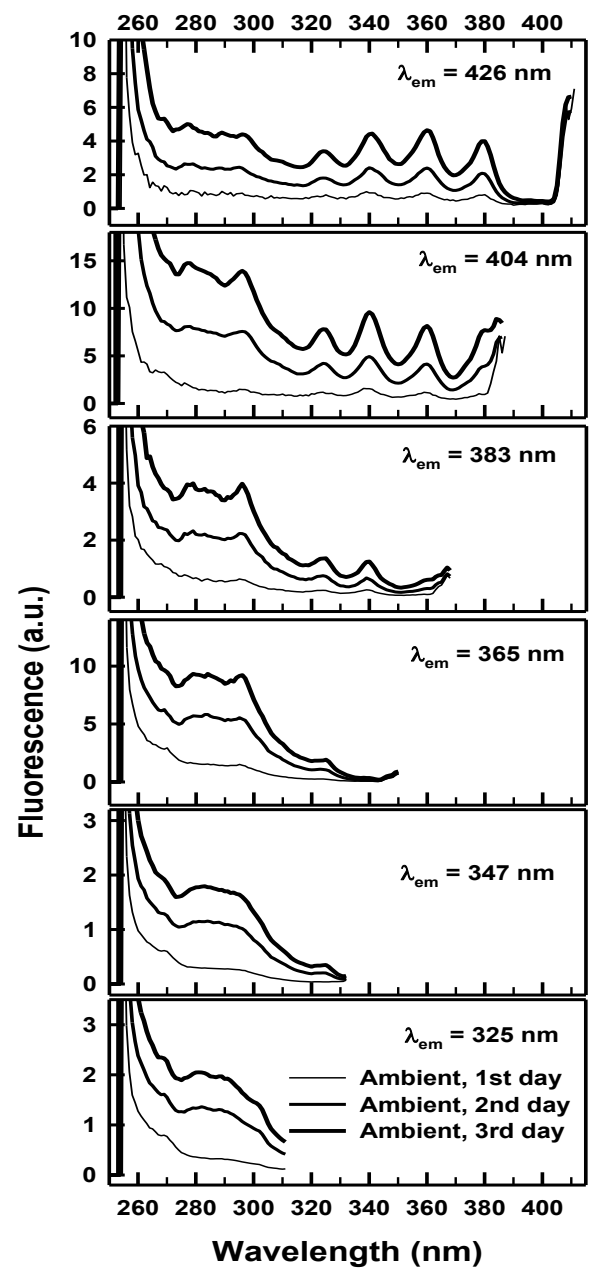

Fig. 10. The excitation spectra at different emissions from PS fine particles under ambient conditions for different days.

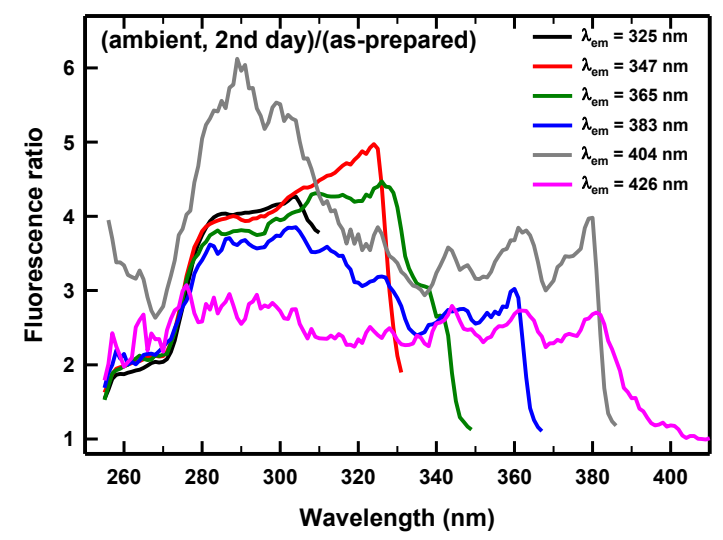

Fig. 11. The ratios of excitation spectra at different emissions from PS fine particles in ambient conditions for one day to the spectra recorded on as-prepared PS particles.

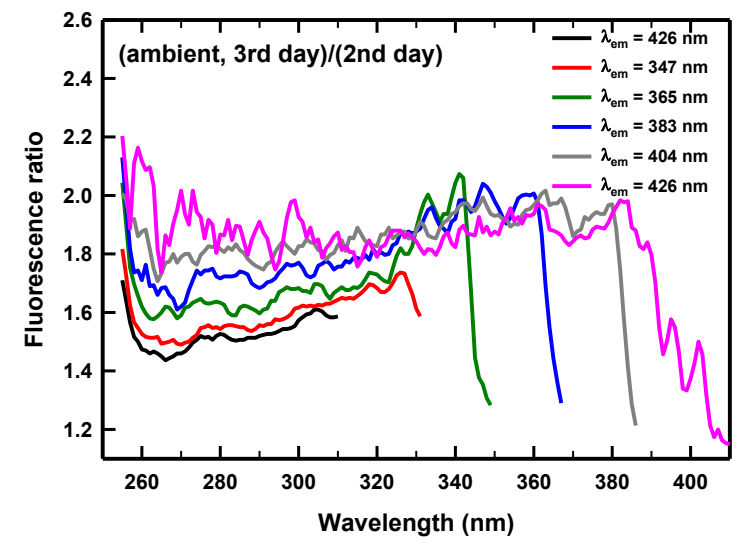

Fig. 12. The ratios of excitation spectra at different emissions from PS fine particles under ambient conditions for 2 days to the spectra from PS particles in ambient conditions for one day. 
The ratios of PS fine particles' excitation spectra after one day's vacuum drying to those before vacuum drying are presented in Fig. 13. The ratio profiles at $\lambda_{\mathrm{em}}=325$ $\mathrm{nm}$ and $\lambda_{\mathrm{em}}=347 \mathrm{~nm}$ are very flat curves with ratios are nearly equal to one. This shows no growths in the fluorophores led to the emissions at $325 \mathrm{~nm}$ and $347 \mathrm{~nm}$, or if any growths exist, their fluorescence emissions will be transferred to other longer wavelengths than 325 and $347 \mathrm{~nm}$.

The ratio profiles at $\lambda_{\mathrm{em}}=365 \mathrm{~nm}$ (in green curve), $383 \mathrm{~nm}$ (blue) and $404 \mathrm{~nm}$ (grey) all have peaks in the longer wavelength regions. This is very different from the situation when PS fine particles are stored under ambient conditions (Fig. 11), where the fluorescence growths are mostly due to the growths of fluorophores with excitation energies in shorter wavelength regions. At $\lambda_{\mathrm{em}}=365 \mathrm{~nm}$, there is relatively very strong peak between $325-348 \mathrm{~nm}$ (centered at $340 \mathrm{~nm}$ ) in the ration profile. This implies that the growth of fluorophores(s) with excitation energies in the region of 325-348 nm contributes to the fluorescence emission growth at 365 $\mathrm{nm}$. These fluorophores can those with direct fluorescence emission at $365 \mathrm{~nm}$ or those that can

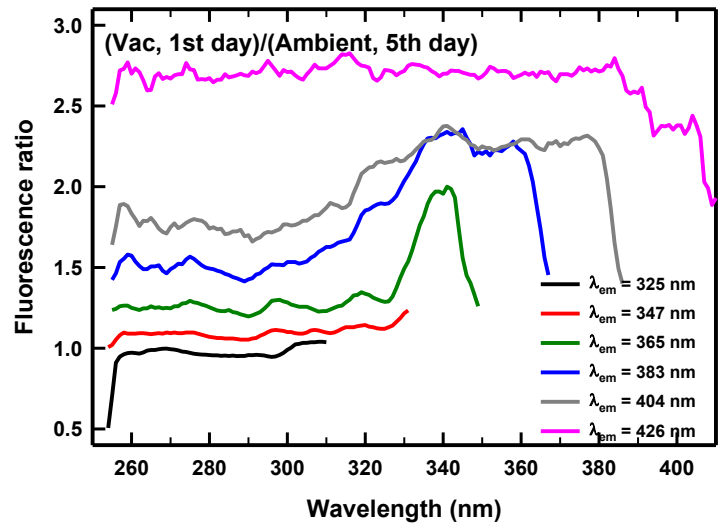

Fig. 13. The ratios of excitation spectra at different emissions from PS fine particles in vacuum for one day to the spectra from PS particles in ambient conditions for 4 days.

As seen in Fig. 9, the PS fine particles after 3 months' vacuum drying show strong growths in fluorescence emissions at 383,404 , and $426 \mathrm{~nm}$ at $\lambda_{\mathrm{ex}}=$ $360 \mathrm{~nm}$. Fig. 15 is their corresponding ratio profiles of the excitation spectra recorded at different fluorescence emissions from the PS fine particles after 3 months' vacuum drying. The ratio profiles at $\lambda_{\mathrm{em}}=383,404$, and $426 \mathrm{~nm}$ all show peaks at $303 \mathrm{~nm}, 321.5 \mathrm{~nm}, 337 \mathrm{~nm}$ and $360 \mathrm{~nm}$. The first three peaks have similar transfer energy to the emission at $365 \mathrm{~nm}$ through absorption and re-emission processes. The fluorescence emission at 383 and $404 \mathrm{~nm}$ shows strong growth in the excitation region of $301-366 \mathrm{~nm}$, and $301-385 \mathrm{~nm}$, respectively. The fluorescence emission at $426 \mathrm{~nm}$ shows the strongest growth in the whole excitation energy range, as indicated by the largest magnitudes in the spectral ratio. However its excitation spectra ratio does not have obvious peaks. This means that excitations along the whole region contribute nearly the same to the growths of the fluorescence emission at 426 $\mathrm{nm}$. The same situation is observed for the emission at $426 \mathrm{~nm}$ when the PS fine particles are vacuum dried for two days, as shown in the purple curve in Fig. 14. In general, the PS fine particles' excitation spectral ratios shown in Fig. 14 share similar profiles as those in Fig. 13, except that the growths in the fluorophores with excitation energy at $296 \mathrm{~nm}$ have relatively stronger contributions to the emissions at $347 \mathrm{~nm}$ and $365 \mathrm{~nm}$ in Fig. 14. As will be discussed in Section 3.3, the contributions of the excitation at $296 \mathrm{~nm}$ to the fluorescence emissions at 347 and $365 \mathrm{~nm}$ are made through energy transfer between different fluorophores.

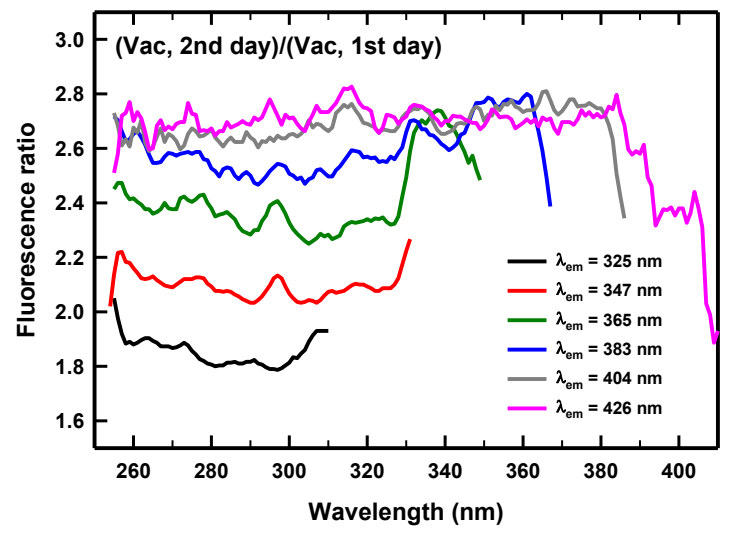

Fig. 14. The ratios of excitation spectra at different emissions from PS fine particles in vacuum for two days to the spectra from PS particles in vacuum for one day.

displacement in their magnitudes in $\mathrm{y}$-axis for the ratio profiles at $\lambda_{\text {em }}=383,404$, and $426 \mathrm{~nm}$, but the latest peak at $360 \mathrm{~nm}$ does not. In other words, when excited at the three peaks' wavelengths, the fluorescence emissions at 383,404 , and $426 \mathrm{~nm}$ will grow at similar rates. However when excited at $360 \mathrm{~nm}$, the fluorescence emissions at 383, 404 and $426 \mathrm{~nm}$ will grow at different rates. This can explain why we observe strong peaks at 383,404 and $426 \mathrm{~nm}$ in the fluorescence ratio profiles at 
$\lambda_{\text {ex }}=360 \mathrm{~nm}$ in Fig. 9. Interesting, after 3 months' vacuum drying, the PS fine particles have little growths in the excitation leading to the excimer emission at 325 $\mathrm{nm}$, as indicated by the relatively very small ratio magnitudes shown by the black curve in Fig. 15 . However, the excitations at 260,278 and $296 \mathrm{~nm}$ all show relative strong growths in the emissions in shorter wavelength regions covered the emission at $325 \mathrm{~nm}$, as shown in Fig.9. This implies that the emissions at 325 $\mathrm{nm}$ are mostly absorbed by other fluorophores and then re-emit at longer wavelengths such as 383, 404 and 426 nm. In conclusion, after 3 months' vacuum drying, the polystyrene chains in PS fine particles are in a network with high-efficient energy transfer paths due to the larger density of fluorophores and closer distances between different fluorophores. This phenomenon can be used to study the chain folding in polystyrene system.

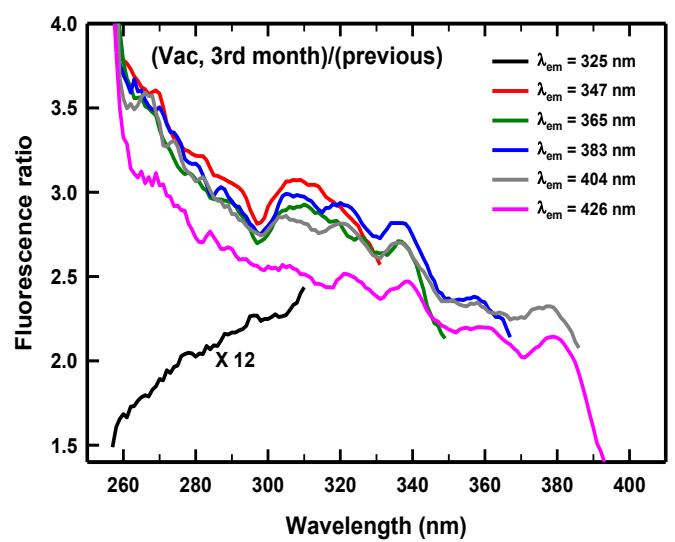

Fig. 15. The ratios of excitation spectra at different emissions from PS fine particles in vacuum for 3 months to the spectra from PS particles in vacuum 2 weeks days before.

\subsection{Energy diagram of excitation/absorp- tion and fluorescence emission bands and related energy transfer.}

Fig. 16 is the plot for the energy difference between observed obvious peaks shown in both the excitation and emission spectra verse the wavelengths of each peak. The plot is based on the spectra recorded on the PS fine particles after 3 weeks' vacuum drying. The emission bands are categorized into three groups, with the emission peaks sandwiched between two parallel lines in three different colors: black, red and green, respectively. The straight lines in different colors connect the fluorescence emissions in different groups categorized with corresponding colors and the absorption peaks shown in their excitation spectra. The excitation of single benzene at $260 \mathrm{~nm}$ can lead to all the fluorescence emissions, therefore the connections to the excitation at $260 \mathrm{~nm}$ are not shown here for simplicity. The connections in solid lines are shared by the whole group of fluorescence emissions, while the connections in dashed lines are specific only for the connected emissions in the group. Heavier lines indicate stronger contributions of the connected absorption peaks to the fluorescence emissions. The excitation/absorption energy at $260 \mathrm{~nm}$ and the emission at $320 \mathrm{~nm}$ are arbitrarily set to zero energy reference points for absorptions and emission bands, respectively.

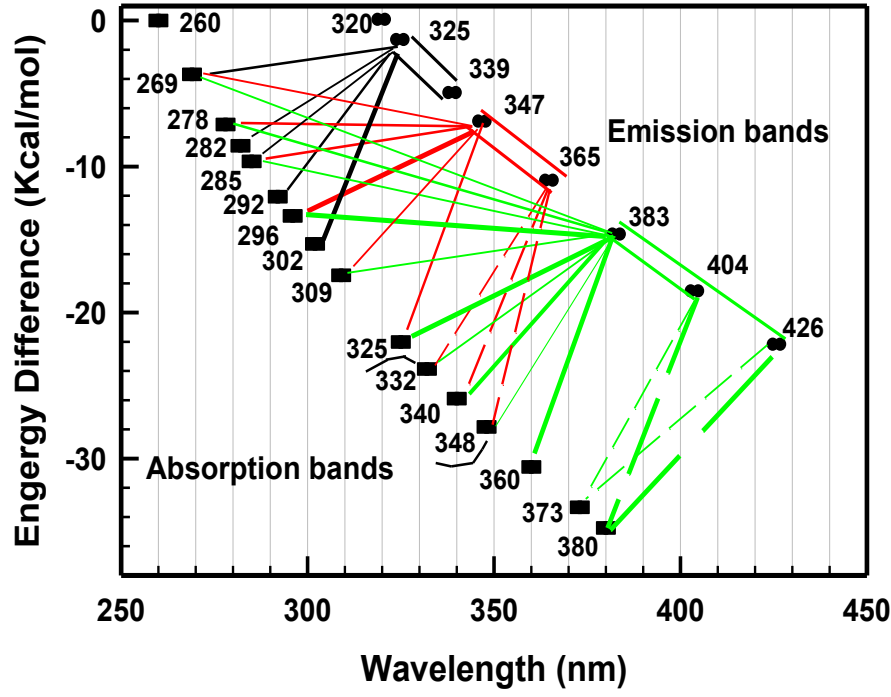

Fig. 16. The energy diagram for the absorption peaks in the excitation spectra and the emission peaks in the emission spectra recorded on PS fine particles after 3 weeks' vacuum drying. See the text for the details about the diagram.

The listed absorption peaks detected in the excitation spectra range from $260 \mathrm{~nm}$ to $380 \mathrm{~nm}$. The energy differences in the unit of $\mathrm{Kcal} / \mathrm{mol}$ for every two adjunct peaks beginning with $269 \mathrm{~nm}$ are $\mathbf{3 . 4 4}(278-269 \mathrm{~nm}), \mathbf{- 1 . 4 6}(282-278 \mathrm{~nm}), \mathbf{- 1 . 0 7}(285-282 \mathrm{~nm}), \mathbf{- 2 . 4 1}$ (292-285 nm),-1.32 (296-292 nm), -1.92 (302-296 nm), -2.15 (309$302 \mathrm{~nm}), \mathbf{- 4 . 5 6}(325-309 \mathrm{~nm}), \mathbf{- 1 . 8 6}(332-325 \mathrm{~nm}), \mathbf{- 3 . 8 8}(340-332$ $\mathrm{nm}), \mathbf{- 3 . 9 6}(348-340 \mathrm{~nm}), \mathbf{- 4 . 6 7}(360-348 \mathrm{~nm}), \mathbf{- 5 . 5 1}(373-360 \mathrm{~nm})$ and -4.18 (380-373 nm), respectively. Besides the excitation of 
benzene at $260 \mathrm{~nm}$, the excitations at 269, 292 and 302 $\mathrm{nm}$ contribute mostly to the emission group of $325 \mathrm{~nm}$ and $339 \mathrm{~nm}$, as indicated by relatively thicker connected lines between them. There is an energy difference of $9.65 \mathrm{Kcal} / \mathrm{mol}$ between the excitation energies of 292 $\mathrm{nm}$ and $269 \mathrm{~nm}$. The large energy difference might indicate the excitation at $292 \mathrm{~nm}$ is to excite the benzene dimer directly, while the excitation at $269 \mathrm{~nm}$ is to excite the benzene monomers in different environments than those with excitation energy at $260 \mathrm{~nm}$. The excited monomer can forms static excimer through interacting with the second phenyl group in place, and then emit emission at 325 and $339 \mathrm{~nm}$. Furthermore, according to the work of Sherrill [3] et al., the binding energies for the sandwich, T-shaped, and paralleldisplaced configurations of benzene dimers are found to be $1.8(2.0), 2.7(2.4)$, and 2.8 (2.7) $\mathrm{kcal} / \mathrm{mol}$, respectively. The binding energies of most and least stable geometries are different by $\sim 1 \mathrm{Kcal} / \mathrm{mol}$. The energy differences between each adjunct absorption peaks between $278 \mathrm{~nm}$ and $302 \mathrm{~nm}$ are listed above. They are some numbers ranging from $-1.07 \mathrm{Kcal} / \mathrm{mol}$ to $-2.41 \mathrm{Kcal} / \mathrm{mol}$. The excitation energy difference of two fluorophores is the sum of energy differences in their ground states and their excited states with vibrational contributions. If two dimers remain similar energy gap in their first electronic excited states as in their ground states, then their excitation energy difference should be around twice of their energy gap in the ground states. Taking all these into account, it is reasonable to consider the absorption peaks between $278 \mathrm{~nm}$ and $302 \mathrm{~nm}$ are attributed to the phenyl dimers in different geometries. Of course, it is also possible that some peaks might correspond to the vibrational structures in the excited state from the same dimer geometry. Consequently, not only the emission at $325 \mathrm{~nm}$ is the excimer emission, but also the emission at $339 \mathrm{~nm}$.

Fluorescence at 347 and 365 are two strong emission peaks (Fig. 1 of previous work [2]). Besides the excitation of benzene monomer at $260 \mathrm{~nm}$, the strongest contributions to the emission at $347 \mathrm{~nm}$ are from the excitation at $296 \mathrm{~nm}$. The excitations at $278 \mathrm{~nm}, 285$ $\mathrm{nm}$, and $325 \mathrm{~nm}$ also contribute to the emission significantly. Interesting, the excitation at $296 \mathrm{~nm}$ does not seem to have obvious contribution to the emissions at either $325 \mathrm{~nm}$ or $339 \mathrm{~nm}$ in the PS fine particles after 3 weeks' vacuum drying. In fact, as shown in Fig. 17, the emissions at excitation energy $296 \mathrm{~nm}$ from the asprepared PS particles (black curve) has major emissions at $339 \mathrm{~nm}$ and $347 \mathrm{~nm}$. However, with enhanced vacuum drying, the emission majors have been shifted to the $347 \mathrm{~nm}$ and $365 \mathrm{~nm}$ (blue and purple curves) through the energy transfer. Correspondingly, the absorption peak at $296 \mathrm{~nm}$ shown in the excitation spectra at $\lambda_{\mathrm{em}}=347 \mathrm{~nm}$ is not very strong when the PS fine particles stay under ambient conditions, but it becomes the strongest peak with further vacuum drying. Therefore, the emission at $339 \mathrm{~nm}$ must be absorbed by fluorophores(s) with emission at $347 \mathrm{~nm}$. As discussed above, the excitations energies of 278-302 nm are considered to belong to the absorption energies of phenyl dimers in different geometries. The energy differences of the absorption peak at $325 \mathrm{~nm}$ from the peak at $302 \mathrm{~nm}$ are $-6.70 \mathrm{Kcal} / \mathrm{mol}$. The big energy differences might indicate that the absorption peak at $325 \mathrm{~nm}$ is from phenyl aggregations with more phenyl groups than two. Therefore the absorption peak is very likely due to the phenyl trimers, and consequently the emission at $347 \mathrm{~nm}$ is the fluorescence emission from phenyl trimer. Why do the excitations of phenyl dimers at 278,285 , and $296 \mathrm{~nm}$ have large contribution to the emission at $347 \mathrm{~nm}$ (trimer)? This is because that the emissions resulting from these excitations of dimers fall into the absorption region of the phenyl trimer with emission at $347 \mathrm{~nm}$. Another possibility is that the excited dimers could interact with an additional phenyl group in the right geometry to form excited phenyl trimers with emission at $347 \mathrm{~nm}$.

The fluorescence emission at $365 \mathrm{~nm}$ shares some common absorption peaks with the emission at $347 \mathrm{~nm}$, but it also has its specific absorption peaks at 332,340 and $348 \mathrm{~nm}$. These three absorption peaks are completely separated in the excitation spectrum at $\lambda_{\mathrm{em}}=$ $365 \mathrm{~nm}$. However, in the excitation spectrum at $\lambda_{\mathrm{em}}=$ $383 \mathrm{~nm}$, the peak at $340 \mathrm{~nm}$ becomes overwhelming

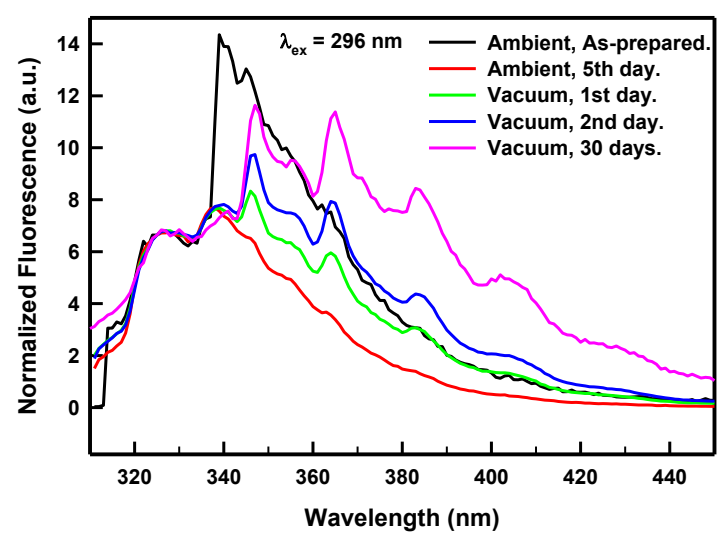

Fig. 17. The normalized fluorescence emission spectra at $\lambda_{\mathrm{ex}}=296 \mathrm{~nm}$ recorded on PS fine particles in different days. 
dominate and the two other peaks at $332 \mathrm{~nm}$ and $348 \mathrm{~nm}$ appear as two small shoulders on the dominate peak at $340 \mathrm{~nm}$. The energy differences between two adjunct absorption peaks of 332, 340 and $348 \mathrm{~nm}$ is around 2 $\mathrm{Kcal} / \mathrm{mol}$. These energy differences are much larger than the calculated vibrational frequencies of trimers [4] Therefore the peaks at 332 and $348 \mathrm{~nm}$ are not likely to be the vibrational structures if the computation results [4] are valid for the polystyrene system.

The energy transfer by absorption of emissions at shorter wavelengths and re-emissions of fluorescence at longer wavelengths occurs more efficiently for the emissions at 383, 404 and $426 \mathrm{~nm}$ (the green group). As indicated by the thick connection lines between the group of 383, 404 and 426 and the absorption bands. The excitations at 278 and $296 \mathrm{~nm}$ (dimers), $325 \mathrm{~nm}$ (trimer), (332, 340 and $348 \mathrm{~nm}$ ) (trimers), and $360 \mathrm{~nm}$ all contribute greatly to the fluorescence emissions at $383-426 \mathrm{~nm}$. The same as the emissions at 347 and 365 $\mathrm{nm}$ (the red group), the emissions in the green group have big contribution from the excitation at $296 \mathrm{~nm}$. This really makes the fluorophores with the excitation energy 296nm special. Different from the emissions in the red group, the absorption peaks at $325 \mathrm{~nm}$ and 340 $\mathrm{nm}$ are very strong in the excitation spectra of the green group emissions. Moreover, there are a strong new absorption peak at $360 \mathrm{~nm}$ shown up for all the emissions in the green group, and another strong new absorption peak at 380 contributed to the emissions at 404 and $426 \mathrm{~nm}$ in the green group. The energy differences between the two adjunct absorption peaks at 325 , $332,340,348,360$ and $380 \mathrm{~nm}$ are $1.86 \mathrm{Kcal} / \mathrm{mol}$, $2.03 \mathrm{Kcal} / \mathrm{mol}, 1.93 \mathrm{Kcal} / \mathrm{mol}, 2.74 \mathrm{Kcal} / \mathrm{mol}$, and 4.18 $\mathrm{Kcal} / \mathrm{mol}$, respectively. The interaction energies of benzene trimer in different geometries are calculated to be $-5.20,-3.33$, and $-3.55 \mathrm{Kcal} / \mathrm{mol}$, respectively [4]. They are different by $0.22 \mathrm{Kcal} / \mathrm{mol}$ to $\sim 2 \mathrm{Kcal} / \mathrm{mol}$. The interaction energies of benzene tetramers in different geometries are calculated to be $-8.55,-7.84$, 7.86, -7.32, $-5.17 \mathrm{Kcal} / \mathrm{mol}$, respectively[4]. They are different by near zero to $-3.4 \mathrm{Kcal} / \mathrm{mol}$. It is hard to predict whether there are some phenyl tetramers contributing to the emissions in the green group based on the energy differences. However, as discussed in Section 3.1, based on the shape of fluorescence profiles, the excitation energies at 360 and 380 seems to be attributed to different fluorophores from those with excitation energies at 325 and $340 \mathrm{~nm}$. It is thus possible that the excitation energies at 360 and $380 \mathrm{~nm}$ could be attributed to the phenyl tetramers. Consequently, the emissions at 383, 404 and $426 \mathrm{~nm}$ could be from phenyl tetramers.
For the emissions in the green group, the two parallel green lines are not in equal lengths as those in red and black. This is because that the excitation at $296 \mathrm{~nm}$ has relatively very strong contribution to the emissions at 383 and $404 \mathrm{~nm}$, but its contribution to the emission at $426 \mathrm{~nm}$ is not that strong. Therefore one of the two parallel green lines does not pass through the data point at $426 \mathrm{~nm}$ to indicate some differences exists there for the emission at $426 \mathrm{~nm}$.

\section{Summary}

The growth of fluorescence emissions reported previously in the polystyrene fine particles [2] under ambient conditions and vacuum drying are explored in greater details. It is found that in the studied system, the fluorescence emissions in longer wavelength regions can be excited in the as-prepared PS samples. The fluorescence growths are presented in the format of normalized emission spectra in different days and in the format of ratio profiles of both emission spectra and excitation spectra. The fluorescence emissions start to grow greatly after one day's stay under ambient conditions allowing the solvent to evaporate freely. The growths are focused on the fluorescence in shorter wavelength regions. The fluorescence growths in the following a few days under ambient conditions are much slower compared to the first day. When drying in vacuum, the PS fine particles exhibit stronger growths for the fluorescence emissions in longer wavelength regions. The strongest growth rates are in the first two days of vacuum drying. After 3 months, the PS fine particles still exhibit obvious fluorescence growths. It is concluded that not only solvent evaporation but also the time are important factors contributed for the chain motions and formations of different fluorophorous phenyl aggregations in the studies polystyrene system, in addition to the aromatic-aromatic interactions and the hydrophobic interactions during reprecipitation that have been discussed previously [2]. It also implies that the polystyrene chains are not in an equilibrium state after staying for 3 months. Besides the local chain motions driven by different forces in the course of solvent removal, other environmental disturbance, such as impacts from excitation light during spectral recordings, and the switching between vacuum storage and the ambient measurements, could also contribute to the non-equilibrium state of the polystyrene system.

There are large overlapping between the absorption bands and emission bands of the polystyrene fine particles. This leads to the efficient energy transfer between different fluorophores. When left in vacuum to 
dry faster and longer, the PS fine particles can form a network with larger density of phenyl aggregations with different and more phenyl groups. This makes the energy transfer between different phenyl aggregations more efficient. An attempt is made to identify the numbers of phenyl groups in the aggregations attributed for the announced emission and absorption peaks based on their energy differences, previous computation studies by other scientists, and their spectral shapes. It is estimated that the absorption peaks centered at 278, 282, 285, 292, 296 and $302 \mathrm{~nm}$ are caused by the phenyl dimers, the absorption peaks centered at $325,332,340$, and $347 \mathrm{~nm}$ are from the phenyl trimers, while the absorption peaks at 360 and $380 \mathrm{~nm}$ are belong to the phenyl tetramers. Consequently, the emissions at 326 and $229 \mathrm{~nm}$ are from the excited phenyl dimers, the emissions at 347 and $365 \mathrm{~nm}$ are from the excited phenyl trimers, while the emissions at 383,404 , and 426 $\mathrm{nm}$ are emit by the excited phenyl tetramers. More proof is needed to support or correct this estimation. For instant, fluorescence lifetime measurements or computation in a polystyrene system could add some constructive information.
The fluorescence phenomena observed in the polystyrene system can be extended to other aromatic macromolecules to study their chain folding, relaxations and other properties.

\section{Acknowledgement}

I want to thank for Susan's invaluable help and suggestions.

\section{References}

[1]Joseph R. Lakowicz, Principles of fluorescence spectroscopy, Volume 1, Springer, 2006.

[2] Kuo A., CheM, 2011, 1, 1-8. http://dx.doi.org/10.5618/chem.2011.v1.n1.1

[3] Mutasem Omar Sinnokrot, Edward F. Valeev, and C. David Sherrill, J. Am. Chem. Soc., 2002, 124 (36), 10887-10893. http://dx.doi.org/10.1021/ja025896h

[4] O. Engkvist and P. Hobza, H. L. Selzle and E. W. Schlag, J. Chem. Phys. 1999, 110, 5758-5762. http://dx.doi.org/10.1063/1.478474 